RELATO DE EXPERIÊNCIA

Recebido em: 21/10/2015

Aceito em: $06 / 03 / 2016$

\title{
Proposição de um índice-h para a produção científica da construção civil nacional
}

\author{
Proposal for an h-index for scientific production of national \\ construction in Brazil
}

Cinthia Luana SIMIONI

Graduanda em Engenharia de Produção (UNOCHAPECO) - cinthialuana@unochapeco.edu.br

Caroline DALLACORTE

Mestranda profissional em Tecnologia e Gestão da Inovação (UNOCHAPECO) carold@unochapeco.edu.br

Claudio Alcides JACOSKI

Professor do Centro de Tecnologia (UNOCHAPECO) - claudio@unochapeco.edu.br

\section{Resumo}

Nos últimos anos, o país vem obtendo um crescente desempenho na produção científica, portanto, é necessário utilizar metodologias para medir a atuação de pesquisadores. A construção civil nacional desempenha um importante papel no desenvolvimento do país e carece de mecanismos de qualificação das ações de P\&D utilizando-se de um dos principais Centros de Referência do Brasil - o infoHab, que possui mais de trinta mil documentos em sua base. Para propor uma metodologia de estruturação do índice-h, testaram-se diversas ferramentas tendo como resultado a criação de um índice-h específico para a construção civil nacional. Esta solução permite um controle da qualidade da produção e volume de citações referenciadas.

Palavras-chave: Produção Científica. Índice-h. InfoHab.

\begin{abstract}
Over the past years, the country has been reaching an increasing performance in scientific production, therefore, it is necessary to use methodologies to measure the action of researchers. The national construction performs an important role in the country development and lacks mechanisms of actions qualification of R\&D using one of the principal reference centers from Brazil - the infoHab, that has more than thirty thousand documents in its base. To propose a methodology of h-índex structuration to construction sector, were tested different kinds of tools having as a result the creation of h-índex specific to national construction. The solution produced allows a quality control of the production and the volume of referenced citations.
\end{abstract}

Keywords: Scientific Production. H-índex. InfoHab.

v. 21, n. 46,2016 p. $137-153$

ISSN 1518-2924
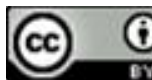

Esta obra está licenciada sob uma Licença Creative Commons. 


\section{INTRODUÇÃo}

O desenvolvimento da produção científica pode ser considerado como um fator chave para sustentabilidade e desenvolvimento do país, uma vez que a atual conjuntura global imprime uma condição de exigência para utilização do conhecimento para geração de novos produtos e processos inovadores para ganho de competitividade.

Este modelo coloca uma certa pressão na produção de conhecimento e na necessidade de qualificação desta produção para posicionamento frente ao mercado. Desta forma, a construção civil que possui uma indústria carente de soluções inovadoras necessita do apoio da academia para avançar na produção técnicocientífica.

O conhecimento e a produção científica são fatores de interesse em todos os países e governos, uma vez que as novas tecnologias e inovações possibilitam um aumento do prestígio e competição para o país, apresentando grandes chances de amplificar a riqueza nacional e o bem estar de seus habitantes.

No Brasil, as agências de fomento utilizam avaliações qualitativas para julgar solicitações de financiamento, que dizem respeito à qualidade intrínseca de seu trabalho e as avaliações quantitativas, retratando a capacidade e regularidade de produção já demonstrada pelo avaliado, considerando ainda, outros aspectos relacionados ao pesquisador ou a instituição (MULLER, 2008).

Nota-se que no ambiente competitivo atual, há um estímulo para que as instituições de pesquisa não fiquem isoladas, mas que apresentem vínculos fortalecidos com o setor produtivo, tornando-se mais relevantes e conseguindo, ao mesmo tempo, mais apoio e recursos. Em países como o Brasil ainda que a vinculação entre a pesquisa científica e o setor produtivo seja importante, o principal parceiro é o setor público (SCHWARTZMAN, 2002).

0 presente trabalho representa, inicialmente, uma importante organização da produção científica para os pesquisadores da construção civil. Muitos projetos já existem no mundo em que se buscam medir em que patamar a produção científica daquele setor se apresenta. A cienciometria e a bibliometria são métodos quantitativos e qualitativos de investigação, que utilizam as referências bibliográficas para avaliar a produção científica, impacto e visibilidade de autores e instituições dentro de uma comunidade científica.

Através de índices como o fator de impacto, é possível mensurar a produção científica de autores que, apesar de possuírem uma produção baixa, desenvolveram material significativo. Estas quantificações estão presentes em diversas bases online, como Scopus, Google Acadêmico, ScienceDirect, além de serem mensuradas através de bases estatísticas como o Journal Citation Reports e Snowball Metrics, que permitem avaliar e comparar publicações, utilizando dados extraídos da comunidade científica para verificar a relevância de um pesquisador, publicação e/ou universidade. As redes sociais para pesquisadores, como ResearchGate e Academia.edu também merecem destaque, uma vez que estimulam o benchmarking e a produção de novas pesquisas.

É dado ênfase ao índice-h, criado pelo professor Jorge E. Hirsch, método utilizado para estimar a produtividade e o impacto do trabalho em uma pesquisa ao mesmo tempo, tendo como base seus artigos mais citados, ganhando destaque devido à possibilidade de utilização de uma única medida para caracterizar o impacto da produção científica, sendo apresentadas suas características, vantagens e limitações (FAUSTO; COSTA, 2008).

Por fim, apresenta-se o desenvolvimento de uma proposta de uso do índiceh para o site do Centro de Referência e Informação em Habitação (infoHab). 0 infoHab é um projeto liderado pela ANTAC (Associação Nacional de Tecnologia do Ambiente Construído) e tem como objetivo facilitar o acesso à informação, 
abordando todos os conteúdos da construção civil e oferecendo produtos e serviços que possam servir de auxílio nas etapas processo/ciclo de geração do conhecimento. Foi criado em 1999, tendo o apoio de diversas universidades parceiras, e atualmente concentra a sua coordenação na Unochapecó, que se encarrega dos aspectos operacionais, logística, e funções técnico-administrativos.

A proposição do índice-h se deu por meio de testes de diversas ferramentas voltadas a identificar a melhor maneira de criá-lo, sendo eles: Mendeley, Altmetric, Oracle e Lucene. Optou-se pela utilização da ferramenta Oracle, onde o processo inclui a busca dos autores cadastrados no infoHab em referências de trabalhos desenvolvidos por outros autores, a fim de identificar qual o impacto de sua produção dentro do próprio site.

\section{2 ÍNDICES DE AVALIAÇÃo DA PRODUÇÃO CIENTÍFICA}

\subsection{Métricas utilizadas para benchmarking da produção científica}

A citação é um conjunto de uma ou mais referências bibliográficas que, sendo incluídas em uma publicação, evidenciam elos entre indivíduos, instituições e áreas de pesquisa, mostrando o relacionamento de uma publicação com outra (ARAÚJO, 2006).

No esforço de descobrir o que já foi produzido cientificamente, a pesquisa bibliográfica assume grande importância, impulsionando o aprendizado, o amadurecimento, os avanços e as novas descobertas nas diferentes áreas do conhecimento. Desta forma, o levantamento bibliográfico na busca do conhecimento tem a intenção de facilitar o caminho percorrido pelo pesquisador até a informação desejada (PIZZANI, 2012).

O saber científico se torna público através das publicações de trabalhos, de forma que as referências bibliográficas tem a função de dar credibilidade para os fatos citados no texto, além de serem utilizadas para dirigir o leitor para as fontes de informação sobre o assunto e também para reconhecimento de um cientista em particular. Assim, uma das obrigações dos pesquisadores é disseminar o conhecimento científico, sendo divulgado os resultados de qualquer investigação de forma a estarem disponíveis para a comunidade, realimentando o processo de comunicação científica (NORONHA, 1998; VANZ, 2003).

0 acesso facilitado à informação por meio das novas mídias está ocupando um espaço cada vez mais privilegiado na construção do saber e na própria formação do indivíduo. A popularização dos computadores e a introdução da internet no âmbito social mostraram grande avanço até então percorrido pela tecnologia da informática digital. Com a web, cresceu o contato do indivíduo com as mais diversas culturas e conteúdos, e as possibilidades de interação homem-máquina e homemhomem, em nível virtual e escala mundial, surgindo novas perspectivas quanto às práticas de comunicação e informação (COSTA et al., 2009).

Para os pesquisadores, uma das vantagens das bibliotecas digitais, é que permite o acesso fácil e rápido no intercâmbio das informações com outras bases de dados e possibilita o trabalho direto com versões eletrônicas de documentos completos (ALISSON, 2015).

Para garantir a integridade dos documentos digitais é preciso armazenar os mesmos em um sistema confiável, assim os repositórios digitais se configuram no âmbito global como a melhor alternativa para a preservação em longo prazo (SANTOS; FLORES, 2015).

Encontram-se ainda, outras formas de avaliação da produção científica, como as medidas bibliométricas, que Vanz (2003) determina como medidas que evoluem ao longo do tempo e que possuem o objetivo de medir o impacto e a visibilidade de determinados autores dentro de uma comunidade científica. 
Entende-se por bibliometria a "técnica quantitativa e estatística de medição dos índices de produção e disseminação do conhecimento científico." (ARAÚJO, 2006, p. 12). Também possibilita a mensuração das fontes de informação utilizadas, como o tipo de documento, o idioma e os periódicos mais citados. Utilizando estes indicadores, é possível saber como se dá a comunicação científica de uma área do conhecimento, obtendo um mapeamento e descobrindo teorias e metodologias consolidadas.

Atrelado a isto, observa-se ainda a métrica da ciência, também conhecida por Macias-Chapula (1998) como cienciometria, sendo denominada como um ramo das ciências da informação, onde uma de suas funções é disseminar conhecimentos, proporcionando informações a respeito da dinâmica, bem como orientação do país, avaliando a sua participação na ciência e na tecnologia mundial.

Outro elemento que apresenta elevada importância é a métrica do Fator de Impacto (FI), que consiste em "dividir o número total de citações obtidas por um periódico em um ano qualquer pelo número de artigos publicados naquele ano." (RODRIGUES, 1981, p. 10). Com este índice, é possível identificar autores que, apesar de possuírem uma produção baixa, desenvolveram material significativo.

\subsection{Modelos mais utilizados atualmente}

\subsubsection{Bases de Dados}

As bases de dados constituem uma importante referência para obtenção de trabalhos científicos, servindo como suporte para os mais variados tipos de pesquisa, pois apresentam um repositório amplo em um único espaço, facilitando a acessibilidade dos usuários.

\subsubsection{Scopus}

Scopus é a maior base de dados do mundo, disponibilizando acesso a resumos e citações de artigos de revistas acadêmicas. Possibilita a identificação de artigos de periódicos em diversas áreas do conhecimento, possuindo ferramentas sofisticadas para rastrear, analisar e visualizar a pesquisa. Identificam-se as publicações mais recentes, mais antigas, ordem decrescente do número de citações ou sua relevância. Existem aproximadamente 19.500 títulos de 5.000 editoras em todo o mundo, também contém mais de 18 milhões de referências a artigos de revistas científicas. (COSTA, 2012).

A base de dados Scopus (Figura 1), indexa as coleções de periódicos brasileiros e de outros países em desenvolvimento da América Latina e Caribe contidas na base Scielo. Esta base aponta que é necessário combinar diversas métricas para avaliar o impacto de uma pesquisa. Na página de detalhes da Scopus, uma barra lateral destaca o número mínimo de métricas significativas, assim o pesquisador consegue avaliar tanto impacto quanto os níveis de engajamento na comunidade, mostrando como os leitores veem o artigo (BEATTY, 2015). 


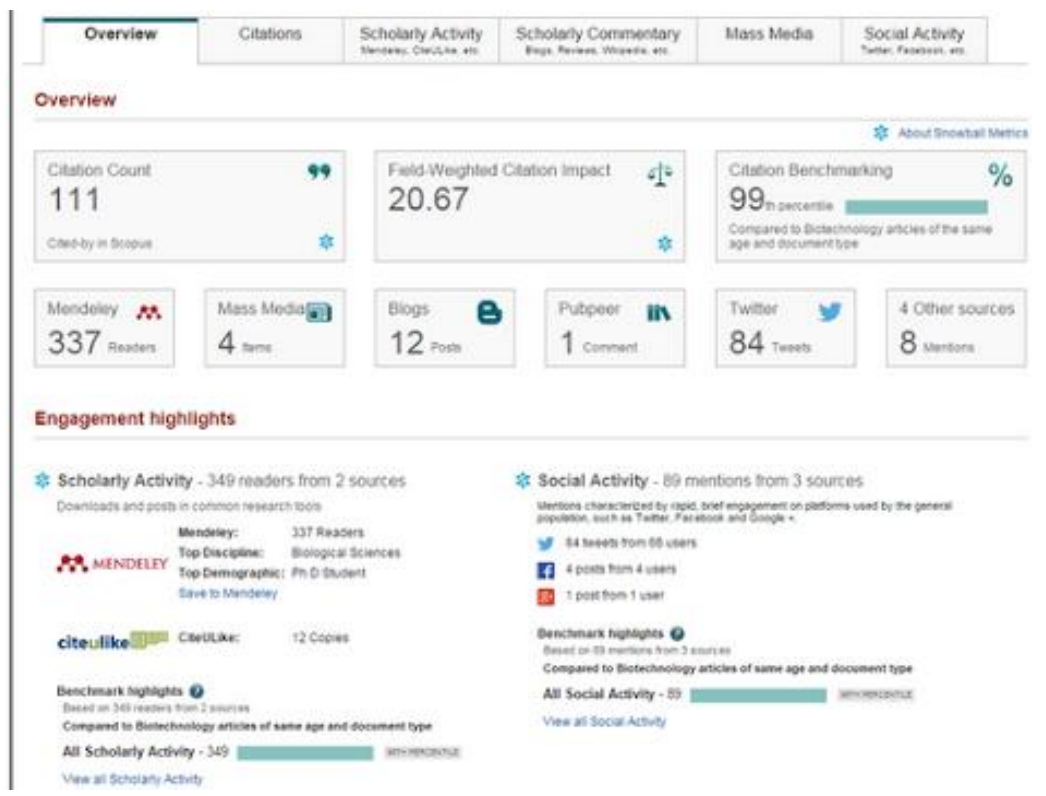

Figura 1: Métricas atuais incorporadas pela base Scopus

Fonte: BEATTY, 2015.

\subsubsection{Google Acadêmico}

O Google Acadêmico permite de maneira simples aos usuários a busca por diversos livros, artigos, teses, resumos e disciplinas de forma abrangente, ajudando ainda a identificar as pesquisas mais relevantes do mundo acadêmico. Os trabalhos são classificados segundo a sua relevância, de forma que a tecnologia de classificação do sistema de buscas, leva em conta o texto integral de cada artigo, o autor, a publicação em que o artigo saiu e a frequência com que foi citado em outras publicações acadêmicas (DÂMASO, 2014).

Com algumas ferramentas disponíveis é possível criar a própria biblioteca, servindo para o internauta guardar artigos e livros de seu interesse facilitando o acesso à textos recorrentes. No campo das métricas pode-se avaliar de maneira prática e rápida a visibilidade e influência de artigos acadêmicos publicados recentemente, o usuário pode explorar as publicações em áreas específicas (DÂMASO, 2014).

Além disso, o Google disponibiliza um espaço destinado a pesquisadores, a fim de estimular o seu reconhecimento e adesão à página. Os autores podem inserir seus trabalhos, e acompanhar através de métricas e citações o impacto das publicações, acompanhando a sua visibilidade como autor. A Figura 2 mostra o perfil geral de um usuário, e um gráfico com a variação de suas citações ao longo dos anos. Também são demonstradas, como o índice-h e o índice i10, métricas responsáveis pela avaliação de produções. 


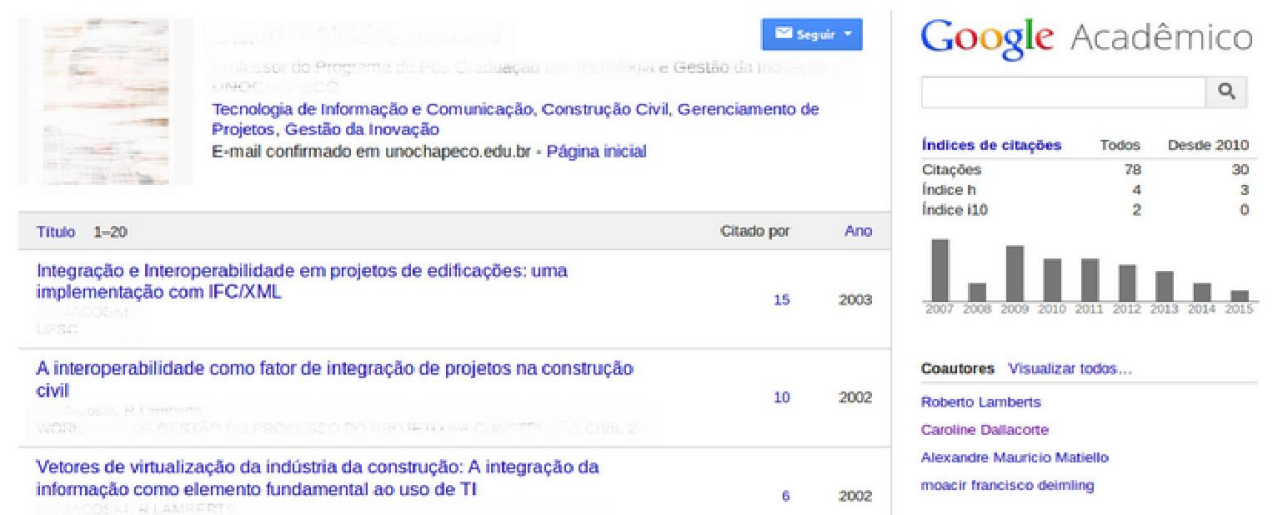

Figura 2: Perfil geral do Google Acadêmico

Fonte: Produzido pelos autores a partir do site Google Acadêmico.

\subsubsection{Institute for Science Information (ISI)}

0 Institute for Science Information da Web of Science, procura suprir as demandas informacionais da comunidade científica nos diferentes campos do saber, onde ajuda a identificar, analisar e compartilhar conhecimento de forma rápida em diversas áreas. Também objetiva prover informações atualizadas e de qualidade aos pesquisadores, oferecendo serviços de banco de dados acadêmicos, com identificação de citações, análise de periódicos e manutenção de bases de dados de citações de milhares de revistas acadêmicas, artefatos de pesquisa, resultados, análise, livros, anais e documentos aprovados (TARGINO; GARCIA, 2000).

Para seleção de revistas são realizadas avaliações qualitativas e quantitativas, nenhum fator é considerado de forma isolada, mas, combinando e inter-relacionando os dados, o editor é capaz de determinar os pontos fortes e fracos de uma revista na sua totalidade, a cada duas semanas, a equipe editorial do ISI analisa cerca de 2 mil títulos novos de revistas anualmente, as revistas atualmente indexadas são monitoradas para assegurar que elas estão mantendo altos padrões e uma clara relevância para produtos do ISI nos quais são repertoriadas (TESTA, 1998).

\subsubsection{SPELL - Scientific Periodicals Electronic Library}

O SPELL é um repositório de artigos científicos com fácil acesso e tem o propósito de promover a organização, disseminação e análise da produção científica de diferentes áreas do conhecimento, possuindo 30.890 documentos. Iniciado em 2012, sua concentração na produção científica se dá nas áreas de administração, contabilidade e turismo, publicadas a partir de 2008, sendo composto por três grandes componentes, os quais são também subprodutos associados ao seu desenvolvimento: Repositório de Artigos Científicos; Sistema de Indexação e Busca de Artigos; Sistema de Informação sobre Periódicos (ANPAD, 2012).

Assim, o SPELL consiste em uma ferramenta virtual que agrega a produção científica disponibilizada eletronicamente pelos periódicos associados, reunindo artigos científicos, resenhas, editoriais, notas bibliográficas, casos de ensino, debates entre outros documentos, onde todos encontram-se livremente disponíveis para consulta e download. Além disso, o SPELL organiza a produção científica oriunda de diferentes periódicos, de modo que proporciona a localização de trabalhos que atendam a um ou vários critérios combinados de busca, os quais podem ser salvos ou compartilhados pelos usuários (ANPAD, 2012). 


\subsubsection{Bases Estatísticas}

Refere-se a bases estatísticas as bases que permitem avaliar e comparar publicações, utilizando dados extraídos da comunidade científica para verificar a relevância de uma publicação, universidade e/ou pesquisador.

\subsubsection{Journal Citation Reports (JCR)}

O Journal Citation Reports fornece um modo simples e objetivo de avaliar os principais periódicos de pesquisa do mundo. Oferecendo uma perspectiva exclusiva para avaliação e comparação de periódicos por meio da acumulação e tabulação de contagens de citações e artigos de praticamente todas as especialidades nos campos da ciência, ciências sociais e tecnologia. Para auxilia-lo a comparar periódicos e descobrir quais são os mais importantes para o pesquisador, o JCR pode mostrar os periódicos mais citados em um campo, periódicos mais importantes, periódicos com maior impacto, artigos mais publicados e dados da categoria do assunto para análise de desempenho (THOMSON REUTERS, 2009).

Usando uma combinação de métricas de impacto e influência, o JCR também fornece o contexto para entender o verdadeiro lugar de uma revista no mundo da literatura acadêmica. Com a facilidade de acesso, podendo personalizar os indicadores e revistas de maior importância para o usuário (THOMSON REUTERS, 2015).

\subsubsection{Snowball Metrics}

O objetivo do Snowball Metrics é compartilhar seus conhecimentos e experiências construindo melhores práticas institucionais baseados em evidências de planejamento estratégico para instituições de ensino superior. As universidades frequentemente lutam para localizar informações de benchmarking confiável, sendo que o Snowball Metrics oferece um ponto de partida para desenvolver significativas comparações que ajudam na tomada de decisões. Assim irá garantir uma definição de metodologias que podem ser aplicadas, a um nível adequado e acordado de detalhe para pesquisa e informação de gestão em todo o setor, quer por instituições, orgãos de financiamento ou fornecedores. Essa consistência facilita a análise comparativa entre as instituições, ajudando a estabelecer uma base confiável para tomada de decisões estratégicas (COLLEGE, 2014).

O Snowball Metrics detalha as metodologias para sua métrica, sendo: produção acadêmica, contagem de citações, índice-h, ponderada pelo campo de impacto de citação, saídas no topo percentis.

\subsubsection{Redes Sociais}

A divulgação de trabalhos acadêmicos atua na formação e conhecimento do pesquisador na comunidade científica. Desta maneira, as redes sociais para pesquisadores estimulam o benchmarking e a produção de novas pesquisas.

\subsubsection{ResearchGate}

O ResearchGate foi criado pelo médico alemão Ijad Madisch em 2008. Tratase de uma rede social livre destinada a integração entre cientistas e pesquisadores de todas as áreas do conhecimento do mundo. A plataforma oferece diversos recursos interativos incluindo compartilhamento de arquivos e de publicações, fóruns, discussões metodológicas, além da busca por resumos das publicações armazenadas no repositório (D'LAMA, 2012).

0 maior benefício do ResearchGate é que os próprios pesquisadores introduzem suas informações curriculares, áreas de pesquisa e temáticas de interesse, assim como suas publicações, de forma compartilhada, aumentando as chances de seus trabalhos serem vislumbrados por colegas com os mesmos interesses. Com a análise das informações fornecidas pelo usuário na página do 
perfil, a plataforma sugere grupos, outros membros e literatura com interesse de pesquisa semelhantes que o usuário pode estar interessado (UFSCAR, 2011).

A Figura 3 mostra o impacto do pesquisador sobre seus trabalhos publicados, onde pode-se ver a quantidade de downloads, visualizações, citações e visitas no perfil.
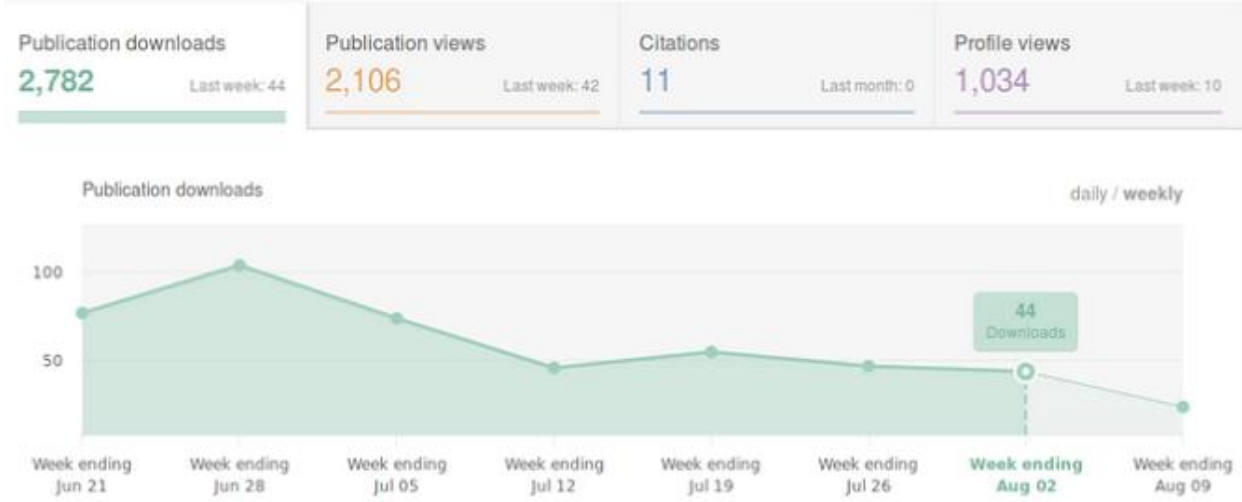

Detailed stats for week ending Aug 022015
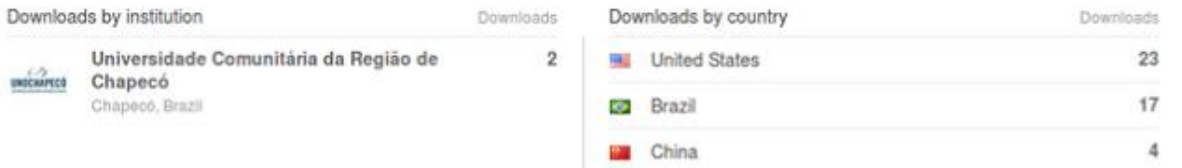

Figura 3: Impacto do pesquisador e suas publicações na rede social ResearchGate Fonte: RESEARCHGATE, 2015.

\subsubsection{Academia.edu}

Academia.edu é uma plataforma com acesso fácil e sem custo para acadêmicos, que somam características de indexador e rede social, permitindo a aproximação de pesquisadores com interesses semelhantes. Como rede social, admite a criação de um perfil que apresente seus interesses de pesquisa e vínculos acadêmicos, publicação de seus trabalhos, poderá também localizar e seguir outros pesquisadores, encontrar e ler publicações de outras pessoas e ver métricas específicas da plataforma que indicam o número de leitores alcançados pelos trabalhos compartilhados no site (THIS, 2014).

\subsection{0 índice-h}

Em 2005, o professor Jorge E. Hirsch da Universidade da Califórnia em San Diego sugeriu a proposição do índice-h, um método simples utilizado para estimar a produtividade e o impacto do trabalho em uma pesquisa, tendo como base os artigos mais citados (FAUSTO; COSTA, 2008). "O índice-h de um pesquisador é definido como o maior número "h" de artigos científicos desse pesquisador que têm pelo menos o mesmo número "h" de citações cada um." (MARQUES, 2013, p. 35). Desta forma, este método vem ganhando destaque devido à possibilidade de utilização de uma única medida para caracterizar o impacto da produção científica.

Para Oliveira e Gracio (2011), algumas características são apresentadas pelo índice como sendo um valor que nunca decresce no decorrer da trajetória de um pesquisador, seu aumento não é linear, pois o indicador não é totalmente influenciado pelo número de trabalhos publicados, mas está fortemente associado ao número de citações e seu valor depende da natureza da área do pesquisador.

Marques (2013) aponta que as principais vantagens do índice-h são voltadas a possibilidade de combinação da quantidade e impacto de pesquisa em um único indicador, a possibilidade de ser obtido por qualquer pessoa com acesso a base de 
dados e pela oportunidade de caracterizar a produtividade cientifica de um pesquisador com objetividade, tendo assim um desempenho melhor do que outros indicadores isolados. Outras vantagens são apontadas por Thomaz, Assad e Moreira (2011), o qual comenta que quanto maior o número de artigos de grande interesse publicado pelo pesquisador, maior será o número de citações alcançadas, e maior será seu índice-h refletindo a qualidade acadêmico-científica do pesquisador e sua capacidade produtiva. Desta forma, pode-se dizer que o índice-h é o resultado do equilíbrio entre o número de publicações e o número de citações. Como exemplo do índice-h pode-se observar em destaque, na Figura 4, o índice-h do Scopus.

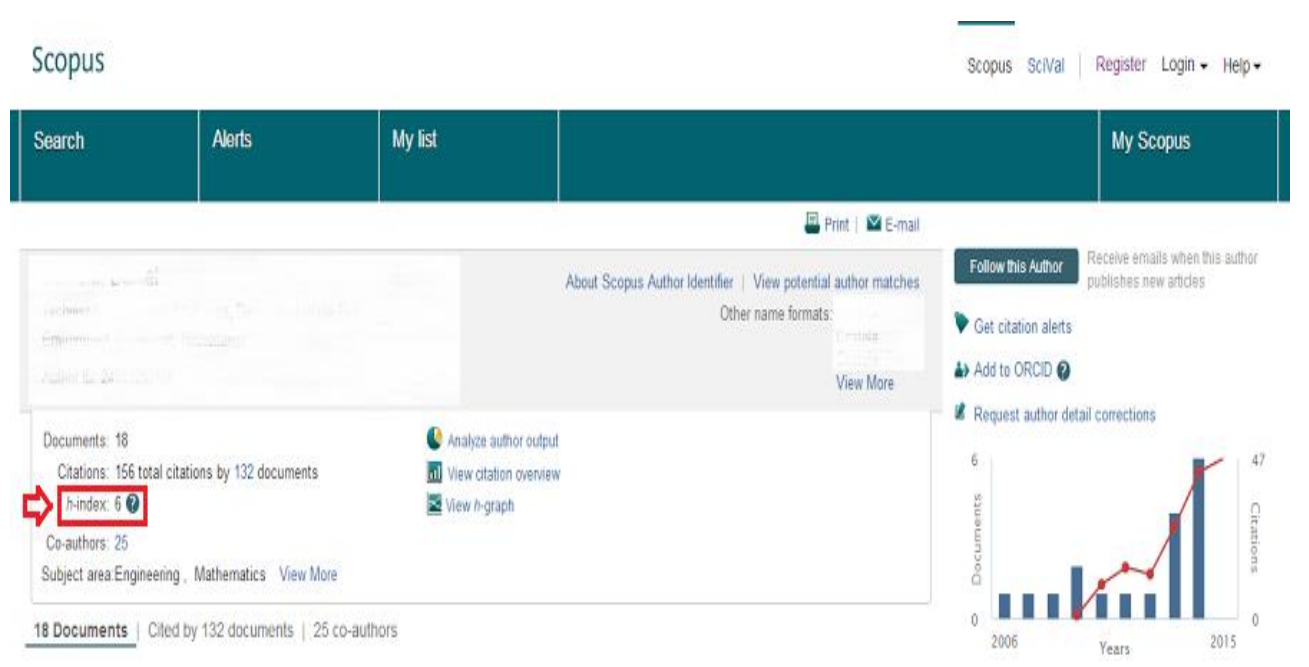

Figura 4: Mostra do índice-h na base Scopus Fonte: SCOPUS, 2015.

Existem algumas desvantagens no uso do índice-h, como: não serve para comparar pesquisadores de disciplinas diferentes; pode ser manipulado por meio de autocitações; dá a livros o mesmo peso que dá aos artigos; não considera o contexto das citações e não faz distinção entre um paper feito por um pesquisador ou um pequeno grupo de colaboradores e um artigo com centenas de autores (MARQUES, 2013).

No Brasil, o índice-h tem sido adotado por orgãos financiadores de pesquisa, de maneira lenta comparado a outros países, mas ajustado por algumas fundações estaduais de amparo à pesquisa (FAPs), pelo Conselho Nacional de Desenvolvimento Científico e Tecnológico (CNPq) e Coordenação de Aperfeiçoamento de Pessoal de Nível Superior (CAPES), como critério para determinar recursos e benefícios, como as bolsas de produtividade científica concedidas pelo CNPq (LIMA; VELHO; FARIA, 2012).

A Plataforma Lattes faz parte do dia-a-dia do trabalho de quem está envolvido na área de pesquisa e docência em instituições de ciência e tecnologia e também nos projetos das principais agências de fomento. 0 acesso ao Lattes pode ser feito por qualquer pessoa, na busca pelo nome, como pode-se ver na Figura 5, mostra o perfil geral do pesquisador, na direita, onde se visualiza o Currículo Lattes, rede de colaboração, diretório de grupos de pesquisa, indicadores de produção e em destaque o link direto do InfoHab, possibilitando assim que todos os dados referentes ao pesquisador possam ser checados. 


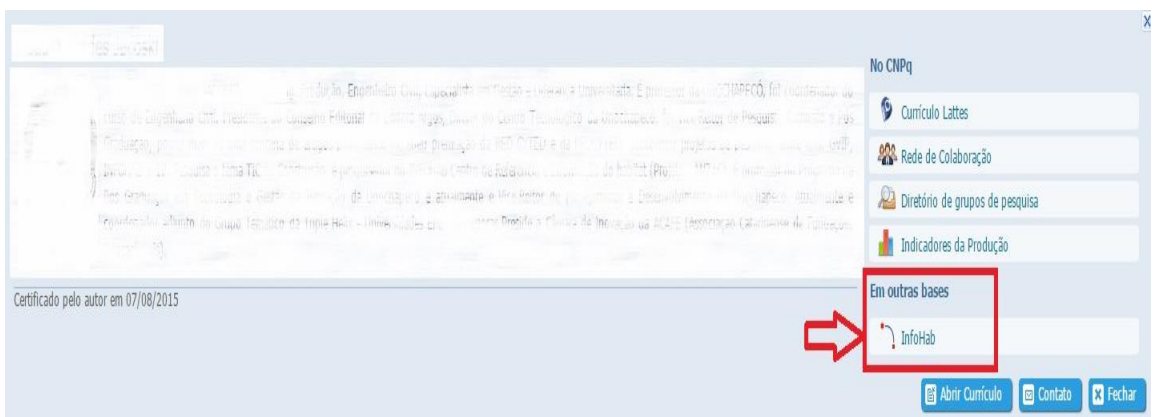

Figura 5: Perfil geral de pesquisador no Lattes, com link direto ao infoHab Fonte: CNPQ, 2015.

A Figura 6, mostra no item produções, onde verifica-se o impacto dos pesquisadores apresentadas pelo Lattes, e apontam o total de citações de cada trabalho nas bases referenciadas.

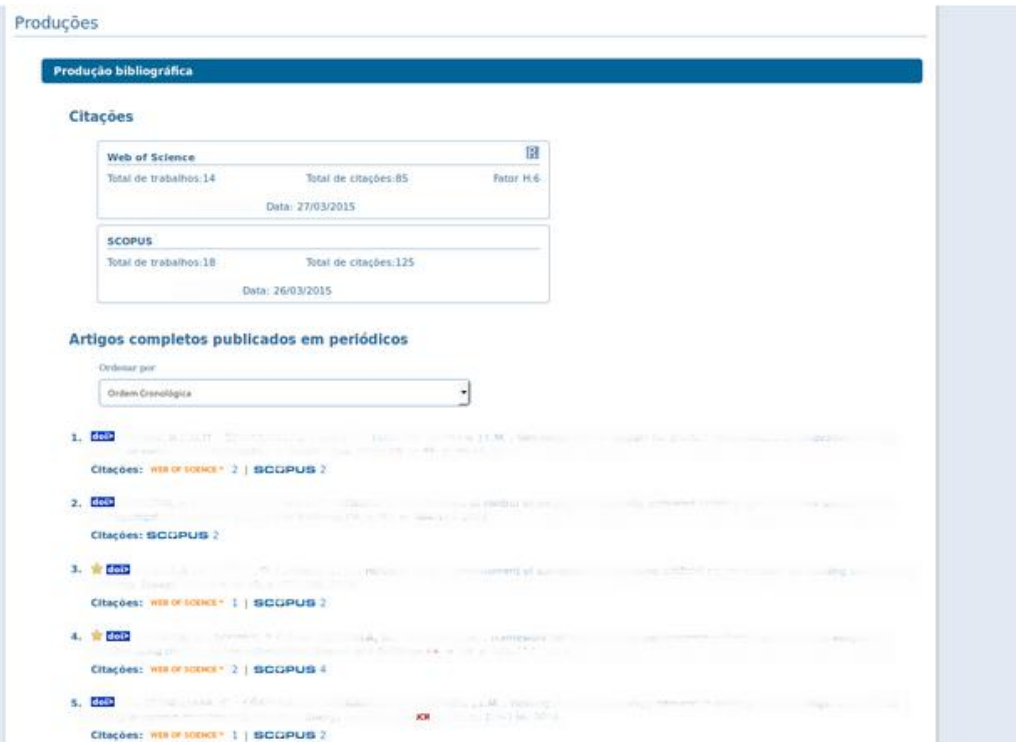

Figura 6: Impacto dos pesquisadores nas bases de dados apresentadas no Lattes Fonte: CNPQ, 2015.

\section{FERRAMENTAS ESTUDADOS PARA PROPOSIÇÃO DA METODOLOGIA}

Para determinar a metodologia de estruturação do índice-h na página do infoHab, foram testadas algumas ferramentas e gerenciadores de referência, a fim de identificar a melhor proposta para o objetivo do trabalho.

\subsection{Mendeley}

O Mendeley é uma ferramenta gratuita, usada como uma rede social acadêmica permitindo a partilha de documentos e a troca de informações, é um gestor de referências bibliográficas para se gerir e citar fontes, gerando a bibliografia de acordo com estilos específicos para publicação, também permite a inserção automática de dados bibliográficos a partir de documentos em formato PDF, podendo ser integrável com os processadores de texto Microsoft Word e Open Office (PEREIRA, 2014).

Com a instalação do Mendeley tem-se outros benefícios, como: Sincronização dos arquivos armazenados no programa; Os arquivos de trabalho gravados tanto nos computadores de uso pessoal quanto na rede; Realizar anotações e marcações 
pessoais nos próprios arquivos, entre outros. Os arquivos no Mendeley podem ser acessados e lidos de forma ampla, sem a necessidade de ajustes no layout do programa (YAMAKAWA et al., 2014).

\subsection{Altmetric}

É uma ferramenta que rastreia o que as pessoas estão dizendo sobre documentos on-line em nome de editores, autores, bibliotecas e instituições, também monitora, pesquisa e mede todas as conversas sobre artigos em jornais, bem como aquelas publicadas por seus concorrentes. Para as instituições, serve para agregar valor a suas bibliotecas e repositórios institucionais. Em pouco tempo se pode trazer dados Altmetric para plataforma revista ou aplicação, como alternativa, se pode escolher apenas as métricas que se achar mais importante (ALTMETRIC, 2015).

\subsection{Oracle Intermedia}

A Oracle é uma empresa de sistemas, que tem como foco o armazenamento de grandes volumes de dados e que trabalha desde o final dos anos 70 no desenvolvimento de softwares de gestão de base de dados. Dentre seus produtos, pode-se citar o Oracle Database, um banco de dados com alto desempenho, que possui uma ferramenta denominada SQL Developer, um cliente gráfico, que tem como funções realizar conexões e operações no banco de dados Oracle. O Oracle SQL Developer é um ambiente de desenvolvimento integrado livre, simplificando o desenvolvimento e a gestão dos dados, tanto em implantações tradicionais ou em nuvem. (ORACLE, 2015).

Através do Oracle Database, pode-se ainda fazer uso do Oracle interMedia, um conjunto de ferramentas que possibilitam o armazenamento e manipulação de dados usados na web como texto, imagens, áudio e vídeo. Também denominada como uma biblioteca, que permite que o Oracle Database armazene, gerencie e recupere informações de localização geográfica, imagens, áudio, vídeo ou qualquer outro dado multimídia de maneira integrada com outras informações da corporação. Além disso, um pool de ferramentas do interMedia trabalha com a indexação e busca de conteúdo web e documentos com formatação Word, PDF, etc (DEVMEDIA, 2015).

Desta forma, através do banco de dados é possível realizar diversas operações necessárias para a implantação de um sistema, unindo qualidade de código e produtividade, essenciais para o desenvolvimento de projetos.

\subsection{Lucene}

O Lucene é uma biblioteca de mecanismo de procura, muito parecido com o Oracle, seu foco principal é na indexação e na procura de texto, uma vez que permite indexar quaisquer dados disponíveis no formato textual. No processo de indexação, armazena os dados de entrada em uma estrutura de dados chamada de índice invertido, que é armazenado no sistema de arquivos ou na memória como um conjunto de arquivos de índice, permitindo que os usuários executem procuras rápidas por palavras-chave e localizem os documentos que correspondem a uma determinada consulta, usando um processo de análise, que é a conversão dos dados de texto em uma unidade de procura fundamental, chamada de termo, os dados de texto passam por várias operações: extração das palavras, remoção de palavras comuns, ignorar pontuação, redução de palavras para o formato de raiz, alteração das palavras para minúsculas, etc. Essa análise acontece imediatamente antes de analisar a indexação e a consulta, convertendo os dados de texto em tokens e esses tokens são incluídos como termos no índice do Lucene (IBM DEVELOPERWORKS, 2015). 


\section{UMA PROPOSIÇÃO DE USO DO ÍNDICE-H PARA O PROJETO INFOHAB}

A fim de identificar a melhor metodologia para utilização do índice-h, fez-se uma análise do uso do Mendeley, verificando que a sua utilização para proposição do índice-h não é viável, uma vez que a extração de informações realizada é específica, fazendo leitura de metadados, como título, autores, páginas e afins, e não de textos do corpo do texto, a fim de identificar as citações ao longo das referências de cada trabalho.

0 retorno do Altmetric, ferramenta utilizada por bases como Scielo, se mostrou novamente não ser pertinente, uma vez que a identificação de citações é realizada apenas em redes sociais, páginas e blogs através de rastreamento, não sendo útil para a proposta em questão.

Buscou-se ainda, avaliar a ferramenta Lucene, que assemelha-se com o objetivo de proposição. Porém, o programa apresenta características muito similares ao Oracle (que é usado como base no projeto infoHab). Sendo assim, decidiu-se testar a ferramenta Oracle para a busca de citações através de consulta no banco de dados.

O objetivo é criar uma proposta de índice-h de autores que possuem trabalhos cadastrados no infoHab, através da busca de citações em trabalhos que estão contidos no acervo. Sendo assim, o primeiro passo foi identificar os autores cadastrados através das ferramentas do próprio sistema gerenciador de banco de dados, o Oracle interMedia, que tem como função realizar a leitura dos pds para verificar quais autores e quantos trabalhos existem na base. Além disso, é utilizado o SQL Developer, que realiza as conexões e operações no banco de dados Oracle. A partir deste, através de processo automatizado, são buscadas as palavras-chave do sobrenome do autor, bem como termos específicos do título de cada trabalho a fim de identificar quantas citações cada um dos trabalhos de cada autor possui no sistema infoHab.

Após esta identificação, é criado um gráfico que apresenta os resultados e pode desta forma calcular o índice-h para cada autor, sendo apresentado junto ao acervo, onde cada trabalho tem link específico que leva as informações do autor contendo seu índice (Figura 7). Além disso, no momento em que o usuário realizar o acesso ao site, será apresentado um ranking de autores com maior índice-h na página principal, conforme mostra a Figura 8, de forma a estimular os autores a enviarem os seus trabalhos para cadastramento. 0 cálculo do índice é realizado através da utilização do método já citado anteriormente. A contagem do índice será realizada pela busca de nome e trabalho de um autor na parte de referências do PDF, através de consultas na base de dados.

\section{Sobre o autor}

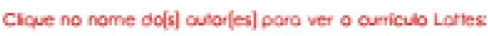

\section{Cláudio Alcides Jacoski}

Documentos 61

Ciloços: 82 totol de citoçoes em $x x$ documentos

Indice he 40

Co-outores: 4

Areos Construçoo civil Gerenciomento de projetos.

Figura 7: Informações sobre o autor

Fonte: elaborado pelos autores. 


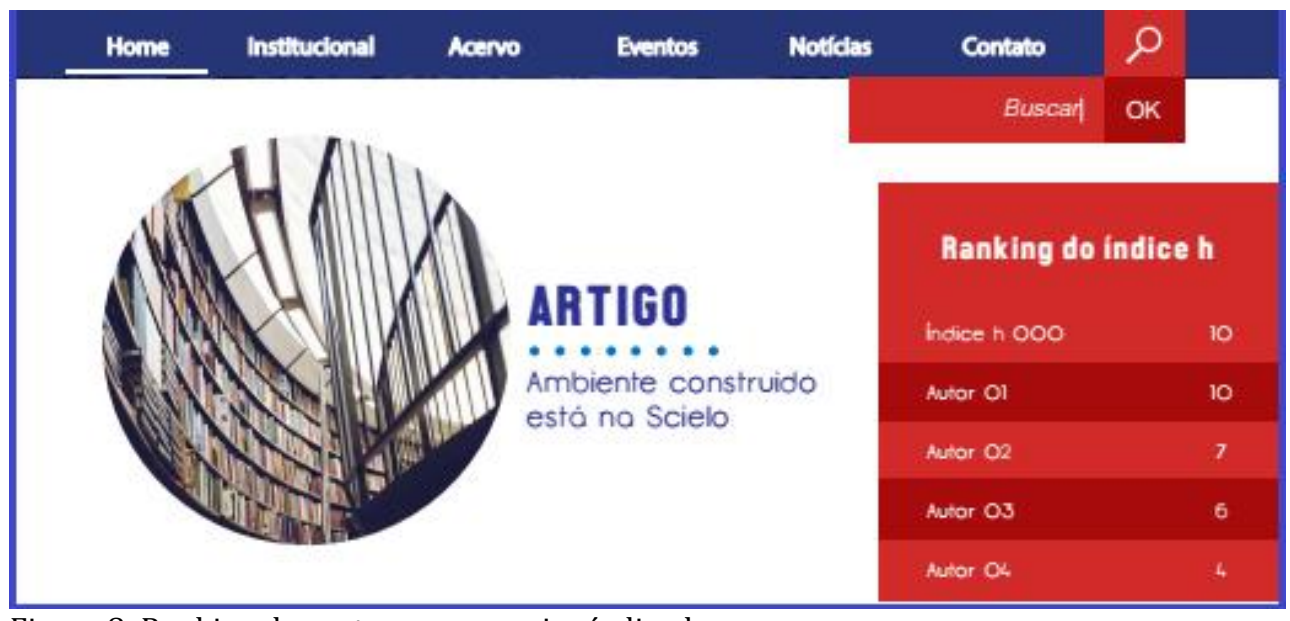

Figura 8: Ranking dos autores com maior índice-h

Fonte: Elaborado pelos autores.

Com a nova proposta no site do infoHab cada autor poderá cadastrar seu trabalho. Da mesma forma, para trabalhos já cadastrados e que não possuem texto completo ou estejam com alguma informação pendente, uma notificação será exibida no momento em que o autor realizar o acesso na página, solicitando as informações que estão em pendência, contribuindo assim para a melhoria do cálculo do índice-h, garantindo que todos os trabalhos citados sejam devidamente referenciados.

Desta forma, espera-se que haja uma maior contribuição dos pesquisadores, aumentando o número de acervos cadastrados, com crescimento de visualizações no site e com a vantagem de aumentar o índice-h dos autores.

\section{CONSIDERAÇÕES FINAIS}

O conhecimento e a produção científica proporcionam o crescimento da competitividade e prestígio dos países, com chances de aumentar a riqueza nacional e o bem estar da população. Nota-se que a utilização da métrica do índice-h para caracterizar produções científicas vem exercendo influência sobre a cultura de publicação de várias áreas do conhecimento, possuindo muitas vantagens e se destacando devido à possibilidade de utilização de uma única medida para caracterizar o impacto da produção científica.

A possibilidade de mensurar a relevância dos pesquisadores da área de construção civil contribui significativamente na valorização de tais investigadores. Desta forma, a proposta apresentada atingiu os objetivos estabelecidos, criando uma proposta de índice-h de autores que possuem trabalhos cadastrados no infoHab, através da busca de citações em trabalhos que estão contidos no acervo do mesmo, uma vez que torna possível medir a quantidade e qualidade da produção científica dos pesquisadores desta área.

Com a reestruturação do site infoHab e a criação do índice-h de cada autor, espera-se que haja maior aproximação dos pesquisadores com a página, incentivando a sua participação e envio de trabalhos, uma vez que almeja-se incorporar este índice ao Currículo Lattes, possibilitando a visualização do impacto destes pesquisadores na página. Portanto, este estudo permite que a comunidade aprofunde ainda mais os conhecimentos a respeito da natureza da produção científica na área da construção civil, apoiando e estimulando a evolução dos pesquisadores. 


\section{REFERÊNCIAS}

ALISSON, E. A expansão das bibliotecas digitais e virtuais. 2015. Disponível em: http://agencia.fapesp.br/a expansao das bibliotecas digitais e virtuais/21753/. Acesso em: 27 ago. 2015.

ALTMETRIC. Who is Altmetric for?. 2015. Disponível em: http://www.altmetric.com. Acesso em: 09 jun. 2015.

ANPAD. Spell - Scientific Periodicals Electronic Library. 2012. Disponível em: http://www.spell.org.br/sobre/objetivos. Acesso em: 31 jul. 2015.

ARAÚJO, C. Bibliometria: evolução histórica e questões atuais. Em Questão, Porto Alegre, v. 12, n. 1, p. 11-32, jan./jun. 2006. Disponível em: http://www.revistas .univerciencia.org/index.php/revistaemquestao/article/viewFile/3707/349. Acesso em: 8 jan. 2015.

BEATTY, S. New Scopus Article Metrics: a better way to benchmark articles, 2015. Disponível em: http://blog.scopus.com/posts/new-scopus-article-metrics-a-betterway-to- benchmark-articles\#VboiW7naxC0.gmail. Acesso em: 6 ago. 2015.

CNPQ. Currículo Lattes. 2015. Disponível em:

http://buscatextual.cnpq.br/buscatextual/busca.do. Acesso em: 17 out. 2015.

COLLEGE, L. Snowball Metrics Recipe Book. 2014. Disponível em:

http://www.snow ballmetrics.com/wp-content/uploads/snowball-recipebook HR.pdf. Acesso em: 6 ago. 2015.

COSTA, A. F. et al. Hipermídia, interface e o novo leitor no contexto digital. 2009. Disponível em:

http://www.revistatxt.teiadetextos.com.br/10/artigo_3_hiermedia_inter face e novo leitor no contexto digital.html. Acesso em: 3 ago. 2015.

COSTA, R. Scopus base de dados. 2012. Disponível em:

http://www.ufrgs.br/gpat/discipl inas/Scopus.pdf. Acesso em: 9 jul. 2015.

DÂMASO, L. Como usar o Google Acadêmico para encontrar artigos científicos online. 2014. Disponível em: http://www.techtudo.com.br/dicas-e-tutoriais/ noticia/2014/03/como-usar-o-google-academico-para-encontrar-artigoscientificos-online.html. Acesso em: 14 out. 2014.

D’ALAMA, L. Rede social para cientistas passa de 1 milhão de seguidores no mundo. São Paulo, 2012. Disponível em: http://g1.globo.com/ciencia-esaude/noticia/2012/01/ rede-social-para-cientistas-passa-de-1-milhao-deseguidores-no-mundo.html. Acesso em: 22 out. 2014.

DEVMEDIA, Full Text Search no Oracle. 2015. Disponível em: http://www.dev media.com.br/full-text-search-no-oracle-parte-01/7793. Acesso em: 13 maio 2015.

FAUSTO, S.; COSTA, F. M. M. O índice-h sob a perspectiva da referência em bibliotecas universitárias: o que os bibliotecários devem saber. In: SEMINÁRIO NACIONAL DE BIBLIOTECAS UNIVERSITÁRIAS, 15, 2008, São Paulo. Anais... São Paulo: CRUESP, 2008. 
GOOGLE ACADÊMICO. Índice de citações. 2015. Disponível em:

https://scholar.google. com.br/citations?user=AlRdbasAAAAJ\&hl=pt-BR. Acesso em: 16 out. 2015.

IBM DEVELOPERWORKS. Usando o Apache Lucene para Procura de Texto. 2015. Disponível em: http://www.ibm.com/developerworks/br/iava/library/os-apachelucen esearch/\#icomments. Acesso em: 13 maio 2015.

LIMA, R. A. de; VELHO, L. M. L. S.; FARIA, L. I. L. de. Bibliometria e "avaliação" da atividade científica: um estudo sobre o índice-h. Perspectivas em Ciência da Informação, v. 17, n. 3, p. 3-17, jul/set. 2012. Disponível em: http://www.scielo.br/scielo.php?script=sci a rttext\&pid=S141399362012000300002. Acesso em: 16 ago. 2015.

MACIAS-CHAPULA, C. A. 0 papel da informetria e da cienciometria e sua perspectiva nacional e internacional. Ciência da Informação, v. 27, n. 2, p. 134-140, maio/ago. 1998. Disponível em:

http://www.tce.sc.gov.br/files/file/biblioteca/o papel da infometria.pdf. Acesso em: 26 jul. 2015.

MARQUES, F. Os limites do índice-h: supervalorização do indicador que combina quantidade e qualidade da produção científica gera controvérsias. 207 ed., maio 2013. Disponível em:

http://revistapesquisa.fapesp.br/2013/05/14/os-limites-do-indice-h/. Acesso em: 6 jan. 2015.

MÜLLER, M. Métricas para a ciência e tecnologia e o financiamento da pesquisa: algumas reflexões. Revista Eletrônica de Biblioteconomia e Ciência da Informação, v. 20, n. 42, p. 24-35, abr. 2008. Disponível em: https://periodicos.ufsc.br/index.php/eb/article/view/15182924.2008v13nesp1p24. Acesso em: 09 abr. 2015.

NORONHA, D. Análise das citações das dissertações de mestrado e teses de doutorado em saúde pública (1990-1994): estudo exploratório. Ciência da Informação, Brasília, v. 27, n. 1, p. 66-75, jan./abr. 1998. Disponível em: http://www.scielo.br/pdf/ci/v27n1/09.pdf. Acesso em: 22 out. 2014.

OLIVEIRA, E.; GRACIO, M. Indicadores bibliométricos em ciência da informação: análise dos pesquisadores mais produtivos no tema estudos métricos na base Scopus. Perspectivas em Ciência da Informação, v. 16, n. 4, p. 16-28, 2011. Disponível em: http://www.scielo.br/scielo.php?pid=S141399362011000400003\&script=sci arttext. Acesso em: 9 jan. 2015.

ORACLE. Oracle SQL Developer. 2015. Disponível em: http://www.oracle.com/technetwo rk/developer-tools/sqldeveloper/overview/index.html. Acesso em: 28 jul. 2015.

PEREIRA, M. de L. dos S. Manual de Utilização do Mendeley. 2014. Disponível em: http://www.miluzinha.com/wp-content/uploads/2011/12/ManualdeInstru\%C3\%A7\%C3 \%B5es-para-a-Utiliza\%C3\%A7\%C3\%A3o-do-Mendeley.pdf. Acesso em: 8 jul. 2015.

PIZZANI, L. et al. A arte da pesquisa bibliográfica na busca do conhecimento. Revista Digital de Biblioteconomia e Ciência da Informação, Campinas, v. 10, n. 1, p. 53- 
66, jul./dez. 2012. Disponível em:

http://www.sbu.unicamp.br/seer/ojs/index.php/rbci/article/v iew/522/pdf 28.

Acesso em: 13 maio 2015.

RESEARCHGATE. Publication downloads. 2015. Disponível em:

http://www.research gate.net/. Acesso: 16 out. 2015.

RODRIGUES, M. P. Estudo das citações constantes das dissertações de mestrado em ciência da informação do IBICT/UFRJ. 1981. Dissertação (Mestrado em Ciência da Informação) - Curso de Pós-Graduação em Ciência da Informação, Instituto Brasileiro de Informação em Ciência e Tecnologia, Universidade Federal do Rio de Janeiro, Rio de Janeiro, 1981.

SANTOS, H. M.; FLORES, D. Repositórios digitais confiáveis para documentos arquivísticos: ponderações sobre a preservação em longo prazo. Perspectivas em Ciência da Informação, Belo Horizonte, v. 20, n. 2, p. 198-218, abr./jun. 2015. Disponível em: file:///C:/Users/usuarios/Downloads/2341-8002-1-PB.pdf. Acesso em: 6 ago. 2015.

SCHWARTZMAN, S. A Pesquisa Científica e o Interesse Público. Revista Brasileira de Inovação, v. 1, n. 2, p. 361-395, jul./dez. 2009. Disponível em: http://ocs.ige.unicamp.br/ojs/rbi/article/view/248. Acesso em: 27 maio 2015.

SCOPUS. 2015. Disponível em: http://www.scopus.com. Acesso em: 15 set. 2015.

TARGINO, M. G.; GARCIA J. C. R. Ciência brasileira na base de dados do Institute for Scientific Information(ISI). Ciência da Informação, v. 29, n. 1, p. 103-117, jan./abr. 2000. Disponível em:

http://www.scielo.br/scielo.php?script=sci arttext\&pid=S010019652000000100011. Acesso em: 26 maio 2015.

TESTA, J.; A base de dados ISI e seu processo de seleção de revistas. Ciência da Informação, v. 27, n. 2, p. 233-235, maio/ago. 1998. Disponível em: http://www.scielo.br/pdf/ci/v27n2/testa.pdf. Acesso em: 6 jul. 2015.

THIS, R. Academia.edu: uma rede social para acadêmicos. 2014. Disponível em: https://bibliotecadafeaacs.wordpress.com/2014/11/03/academia-edu-uma-redesocial-para-academicos/. Acesso em: 10 ago. 2015

THOMSON REUTERS. Journal citation reports: suportado pelo ISI web of knowledge. 2009. Disponível em:

https://www.periodicos.capes.gov.br/images/documents/jcr_qrc_pt_20

101026.pdf. Acesso em: 11 fev. 2015.

THOMSON REUTERS. Journal citation reports: Streamlined. Integrated.

Authoritative, 2015. Disponível em: http://about.jcr.incites.thomsonreuters.com/. Acesso em: 6 jul. 2015.

THOMAZ, P. G.; ASSAD, R.; MOREIRA, L. F. P. Uso do Fator de impacto e do índice-h para avaliar pesquisadores e publicações. Arquivos Brasileiros de Cardiologia, São Paulo, v. 96, n. 2, p. 90-93, fev. 2011. Disponível em:

http://www.scielo.br/scielo.php?script=sci arttext\&pid=S0066-

782X2011000200001\&lng=pt\&nrm=iso\&tlng=pt. Acesso em 15 nov. 2014. 
UFSCAR. ResearchGate: uma rede social para cientistas. 2014. Disponível em: http://www .sea bd.bco.ufscar.br/bases-dados/researchgate-uma-rede-social-paracientistas. Acesso em: 22 out. 2014.

VANZ, S.; CAREGNATO, S. Estudo de citações: uma ferramenta para entender a comunicação. Em Questão, Porto Alegre, v. 9, n. 2, p. 295-307, jul./dez. 2003.

Disponível em: http://seer.ufrgs.br/index.php/EmQuestao/article/view/75/35. Acesso em: 11 out. 2014.

YAMAKAWA, E. K. et al. Comparativo dos softwares de gerenciamento de referências bibliográficas: Mendeley, EndNote e Zotero. Transinformação, Campinas, v. 26, n. 2, p. 167-176, maio/ago. 2014. Disponível em:

http://www.scielo.br/pdf/tinf/v26n2/0103-3786-tinf-26-02-00167.pdf. Acesso em: 8 jul. 2015.

Editores do artigo: Adilson Luiz Pinto e Rafaela Paula Schmitz 